\title{
Función endotelial y lipemia postprandial en adultos con presencia de criterios asociados a síndrome metabólico: efecto del estado nutricional
}

\author{
Diana Milena Díaz-Vidal ${ }^{a}$, Luis Andrés Téllez-T ${ }^{\mathrm{b}}$, Diana Camelo-Prieto ${ }^{\mathrm{b}}$, \\ Alejandra Tordecilla-Sanders ${ }^{a}$, Paula Andrea Hernández-Quiñónez ${ }^{a}$, \\ Esteban Sabogal $^{\mathrm{b}}$, David Chaparro ${ }^{\mathrm{b}}$, Jorge Enrique Correa-Bautista ${ }^{\mathrm{a}}$ \\ y Robinson Ramírez-Vélez ${ }^{\mathrm{a}, *}$
}

\begin{abstract}
a Centro de Estudios para la Medición de la Actividad Física. Escuela de Medicina y Ciencias de la Salud, Universidad del Rosario, Bogotá, Colombia

b Grupo GICAEDS, Facultad de Cultura Física, Deporte y Recreación, Universidad Santo Tomás, Bogotá, Colombia
\end{abstract}

Recibido el 30 de junio de 2016; aceptado el 20 de agosto de 2016

Disponible en Internet el 24 de octubre de 2016

\section{PALABRAS CLAVE \\ Endotelio; \\ Factores de riesgo \\ cardiovascular; \\ Obesidad}

\begin{abstract}
Resumen
Introducción: La lipemia postprandial se caracteriza por un aumento de las lipoproteínas ricas en triglicéridos y varios trabajos lo describen como un factor que determina la salud metabólica de un individuo. El objetivo del estudio fue cuantificar la contribución del sobrepeso en la magnitud de la lipemia postprandial en 33 sujetos con criterios asociados al síndrome metabólico $(n=20$, sobrepeso y $n=13$ eutróficos, $66 \%$ hombres, edad media 31,2 $\pm 7,6$ años).

Métodos: Estudio descriptivo y transversal. Se midió la vasodilatación mediada por el flujo, la velocidad de onda del pulso, el perfil lipídico, el cociente Log triglicéridos/lipoproteínas de alta densidad, la glucosa y la presión arterial tras una ingesta estándar alta en lípidos (79\% $\mathrm{Kcal} /$ grasa). Se calculó el Z-score de riesgo cardiovascular a partir de la suma de los residuos tipificados (Z) de las variables bioquímicas. El estado de lipemia se midió en ayuno ( 0 min.) y a los $(60,120,180$, y 240 minutos) postprandiales.

Resultados: El valor basal de la vasodilatación mediada por el flujo y la velocidad de onda del pulso fue de $6,9 \pm 5,9 \%$ y $7,0 \pm 0,8 \mathrm{~m} / \mathrm{s}$, respectivamente. Se identificó que la lipemia postprandial reducía la vasodilatación mediada por el flujo en $19,2 \%$ a los 60 minutos $(5,9 \pm 1,5 \%$ y a los 240 minutos $(3,7 \pm 1,2 \%)(p=0,04)$, respectivamente. Este hallazgo se acompañó con un aumento en la velocidad de onda del pulso $(p<0,05)$. Al dividir los sujetos en dos grupos según el índice de masa corporal, los participantes en sobrepeso muestran cifras más elevadas en el Zscore de riesgo cardiovascular, la velocidad de onda del pulso, el Log triglicéridos/lipoproteínas de alta densidad y el $\Delta$-velocidad de onda del pulso, $(\mathrm{p}<0,001)$.
\end{abstract}

\footnotetext{
* Autor para correspondencia.

Correos electrónicos: robin640@hotmail.com, robinsonramirez@usantotomas.edu.co (R. Ramírez-Vélez).
} 
Conclusión: Este estudio demuestra que los sujetos clasificados en sobrepeso y que presentan criterios asociados al síndrome metabólico, muestran un perfil cardiometabólico asociado con un mayor riesgo cardiovascular, tras una ingesta alta en grasas.

(c) 2016 Sociedad Colombiana de Cardiología y Cirugía Cardiovascular. Publicado por Elsevier España, S.L.U. Este es un artículo Open Access bajo la licencia CC BY-NC-ND (http:// creativecommons.org/licenses/by-nc-nd/4.0/).

\section{KEYWORDS}

Endothelium; Cardiovascular risk factors; Obesity

\section{Endothelial function and postprandial lipemia in adults presenting criteria associated to metabolic syndrome: effect of nutritional state}

\begin{abstract}
Introduction: Postprandial lipemia is characterised by an increase in triglyceride-rich lipoproteins and several studies describe it as a factor that determines metabolic health of an individual. The motivation of the study was to quantify the contribution to overweight in the magnitude of the postprandial lipemia in 33 persons with criteria associated to metabolic syndrome $(n=20$ being overweight and $n=13$ eutrophic, 66\% male, average age $31.2 \pm$ 7.6 years).

Methods: Cross-sectional descriptive study. Flow-mediated vasodilation, pulse wave velocity, lipid profile, log of the triglyceride/protein ratio, glucose and blood pressure were measured after high standard intake of lipids (79\% Kcal/fat). Z-score for cardiovascular risk was calculated using the sum of typified residues $(Z)$ of biochemical variables. Lipemia state was measured at fasting $(0 \mathrm{~min})$ and after $60,120,180$ and 240 postprandial minutes.

Results: Baseline flow-mediated vasodilation and pulse wave velocity values were $6.9 \pm 5.9 \%$ and $7.0 \pm 0.8 \mathrm{~m} / \mathrm{s}$, respectively. It was identified that postprandial lipemia reduced flowmediated vasodilation by $19.2 \%$ after 60 minutes $(5.9 \pm 1.5 \%)$ and after 240 minutes $(3.7 \pm 1.2 \%)$ $(p=0.04)$, respectively. This finding was paired with an increase in pulse wave velocity $(p<0.05)$. When dividing subjects into two groups according to their body mass index, overweight participants show higher Z-score cardiovascular risk values, pulse wave velocity, log of the triglyceride/protein ratio and the $\Delta$ pulse wave velocity $(p>0.001)$.

Conclusion: This study reveals that overweight individuals showing criteria associated to metabolic syndrome have a cardiometabolic profile linked to a higher cardiovascular risk, after high intake of lipids.

(C) 2016 Sociedad Colombiana de Cardiología y Cirugía Cardiovascular. Published by Elsevier España, S.L.U. This is an open access article under the CC BY-NC-ND license (http:// creativecommons.org/licenses/by-nc-nd/4.0/).
\end{abstract}

\section{Introducción}

La lipemia postprandial se caracteriza por un aumento de las lipoproteínas ricas en triglicéridos que, con una duración de 6 a 10 horas, se produce después de las comidas ${ }^{1}$. En esta condición dinámica pasa la especie humana la mayor parte de su tiempo y varios estudios relacionan este estado metabólico con un incremento en la capacidad aterogénica ${ }^{2-7}$. En estudios experimentales en humanos, se ha observado que el consumo de ácidos grasos saturados y ácidos grasos trans actúan negativamente sobre la función endotelial ${ }^{3}$, princi- $^{-}$ palmente, a través del aumento de las concentraciones de las moléculas de adhesión vascular y de hormonas como la insulina que favorecen el proceso aterosclerótico, así como reduciendo la capacidad de vasodilatación arterial, medida empleada tradicionalmente para conocer la funcionalidad del endotelio ${ }^{4}$. Estos resultados se han corroborado posteriormente en estudios observacionales, lo cual apoya los datos descritos previamente ${ }^{5}$. Además, el colesterol unido a las lipoproteínas de baja densidad y los triglicéridos en los adultos jóvenes predice la aparición de la enfermedad cardiovascular en una fase posterior de la vida ${ }^{6}$, lo cual respalda la idea de que se debe considerar la relación entre las lipoproteínas de baja densidad y los triglicéridos como indicador temprano de enfermedad cardiometabólica ${ }^{7}$.

Por otro lado, el síndrome metabólico es un grupo de trastornos cardiometabólicos considerado como un predictor de la enfermedad cardiovascular, la diabetes mellitus tipo 2 y la mortalidad en general ${ }^{7}$. La definición de síndrome metabólico es objeto de controversia, si bien todas las definiciones vigentes incluyen: la resistencia a la insulina, la inflamación de bajo grado, la disglicemia, la disminución de los niveles plasmáticos en las lipoproteínas de alta densidad, el aumento de los niveles de triglicéridos, la presión arterial elevada, y la obesidad central ${ }^{7}$. En complemento a lo anterior, las alteraciones del estado nutricional como el sobrepeso y la obesidad se asocian directamente con perfiles aterogénicos con la consecuente disfunción endotelial y la liberación de colesterol al espacio subendotelial ${ }^{8}$. En los sujetos normolipídicos (45 mujeres premenopáusicas y 31 varones, edad media 34 años), González y Cols. ${ }^{9}$ mostraron que los sujetos con obesidad abdominal presentaron 
valores mayores de los triglicéridos plasmáticos, de la glicemia y del índice HOMA, con valores plasmáticos bajos del colesterol unido a las lipoproteínas de alta densidad en ayunas luego de un estado de lipemia postprandial, medida con la autodeterminación diurna de los triglicéridos en la sangre capilar. Esta misma observación fue también descrita por Van Wijk y Cols. ${ }^{10}$ en los sujetos obesos con y sin diabetes mellitus tipo 2 y en las personas con normopeso (considerando la obesidad un índice de masa corporal de $27 \mathrm{~kg} / \mathrm{m}^{2}$ o mayor). Estos autores observaron que el perfil diurno de los triglicéridos en la sangre capilar era, significativamente, mayor en el grupo de sobrepeso, sin diferencias entre los diabéticos y los no diabéticos. En este sentido, es posible afirmar que las alteraciones en el estado nutricional se asocia con lipemia postprandial elevada, siendo probablemente, un factor de riesgo cardiovascular encubierto, aun en ausencia de la hipertrigliceridemia en ayunas ${ }^{11}$.

Así pues, el estudio de la lipemia postprandial en presencia de la obesidad en sujetos normolipídicos en las poblaciones de Europa ${ }^{9,10,12}$ o América del Norte ${ }^{4,6,13}$ son abundantes, pero hay pocos datos del efecto de estos parámetros en la lipemia postprandial en poblaciones del Sur de América, que poseen diversos hábitos dietéticos, prevalencias e incidencias de la enfermedad cardiovascular ${ }^{14}$.

El objetivo del presente estudio fue cuantificar la contribución del sobrepeso en la magnitud de la lipemia postprandial en sujetos que cumplen criterios asociados al síndrome metabólico de Bogotá, Colombia.

\section{Materiales y métodos}

\section{Diseño y población}

Durante el primer semestre del año 2015, se planteó un estudio descriptivo y transversal, en 22 hombres y 13 mujeres residentes en el área metropolitana de Bogotá, Colombia. Los criterios de inclusión fueron: a) Presencia de al menos dos componentes del síndrome metabólico: obesidad central (circunferencia de cintura $\geq 90 \mathrm{~cm}$ (hombres) $\geq 80 \mathrm{~cm}$ (mujeres), $\mathrm{y} / \mathrm{o}$ triglicéridos $\geq 150 \mathrm{mg} / \mathrm{dL}$, $\mathrm{y} / \mathrm{o}$ lipoproteínas de alta densidad $<40 \mathrm{mg} / \mathrm{dL}$ en hombres $\mathrm{y}<50$ en mujeres, $y / o$ presión arterial $\geq 130 / 85 \mathrm{~mm} \mathrm{Hg}, y /$ o glucosa plasmática en ayunas $\geq 110 \mathrm{mg} / \mathrm{dL}$; b) Ausencia de antecedentes personales o familiares de dislipidemia, cardiopatía isquémica temprana o diabetes mellitus; c) Índice de masa corporal inferior a $30 \mathrm{~kg} / \mathrm{m}^{2}$ y d) edad entre 20 y 45 años. La selección de la muestra se realizó mediante convocatoria voluntaria. Se excluyeron los participantes con diagnóstico médico de hipertensión arterial, hipo/hipertiroidismo, antecedentes de historia de abuso de drogas o alcohol, medicación hipolipemiante o ingesta de fármacos capaces de modificar el perfil lipídico 4 semanas antes del estudio, dietas hipocalóricas para adelgazar, y padecimiento de procesos inflamatorios o infecciosos. El cálculo del tamaño muestral se realizó asumiendo una diferencia superior al $20 \%$ en los parámetros que evalúan la lipemia postprandial (promedio de puntación de área bajo la curva de los triglicéridos y el colesterol total) entre los determinates a priori conocidos: el sexo y la obesidad. Se fijó el error alfa en un $5 \%$ y el error beta en un $20 \%$. Se obtuvo el consentimiento informado por escrito de cada participante y el Comité de Ética en Humanos del centro académico aprobó la intervención siguiendo las normas deontológicas reconocidas por la Declaración de Helsinki y la normativa legal vigente colombiana que regula la investigación en humanos (Resolución 008430 del Ministerio de Salud de Colombia, acta de aprobación $\mathrm{N}^{\circ}$ 01-1802-2013). Los participantes que aceptaron y firmaron el consentimiento informado se citaron para los siguientes procedimientos:

\section{Evaluación antropométrica, estado nutricional y composición corporal}

Los participantes aportaron información acerca de su historia clínica, donde indicaron antecedentes personales y familiares de: la enfermedad cardiovascular precoz, el hábito tabáquico (número de cigarrillos/día), el consumo de alcohol (gramos de alcohol/día), el ejercicio físico (minutos/semana) y los fármacos de uso habitual u ocasional que coincidiesen con la fecha del estudio. Posteriormente, una valoración antropométrica que comprendió la medición de altura, el peso, la circunferencia de la cintura y la cadera fue realizada a partir de los protocolos estandarizados de López y Cols. ${ }^{15}$, en la población colombiana. La altura se registró en estiramiento con Estadiómetro Portátil (SECA 206 ${ }^{\circledR}$; Hamburgo, Alemania) (rango 0-220 cm) de $1 \mathrm{~mm}$ de precisión. El peso se midió con la balanza de piso SECA mBCA $515^{\circledR}$ (Hans E. Rüth S.A., Hamburgo, Alemania) con capacidad máxima de $200 \mathrm{~kg}$ y mínima de $100 \mathrm{~g}$. Con estas variables se calculó el índice de masa corporal en $\mathrm{kg} / \mathrm{m}^{2}$, y la población se clasificó en dos grupos: el normopeso (índice de masa corporal entre 18,5 y $25,0 \mathrm{~kg} / \mathrm{m}^{2}$ ) y el sobrepeso (índice de masa corporal entre 25,1 y $\left.30,0 \mathrm{~kg} / \mathrm{m}^{2}\right)^{16}$. Posteriormente, con cinta métrica plástica con una precisión de $1 \mathrm{~mm}$ (Holtain Ltd., Crymych Dyfed, RU) se midió la circunferencia de cintura y cadera tomando los referentes anatómicos descritos por la Organización Mundial de la Salud ${ }^{16}$. La relación cinturaestatura fue determinada por la división de la circunferencia de cintura $(\mathrm{cm})$ por la altura $(\mathrm{cm})$. La composición corporal se estimó por bioimpedancia eléctrica de 8 segmentos marca SECA mBCA $515^{\circledR}$ (Hans E. Rüth S.A., Hamburgo, Alemania) de acuerdo con las indicaciones y ecuaciones señaladas en el manual del usuario. La frecuencia de inducción se valoró a una intensidad de $50 \mathrm{kHz}$, con una sensibilidad de estimación de la masa de grasa de $0,1 \mathrm{~kg}(0,1 \%)$. Con este analizador se obtuvo la grasa corporal (\%) y la masa grasa $(\mathrm{kg})$. Esta medición se realizó luego de 10-12 horas de ayuno, con la vejiga vacía y sobre una superficie no conductora.

\section{Biomarcadores metabólicos y otros relacionados a la enfermedad cerebrovascular}

Las mediciones bioquímicas se realizaron tomando una muestra capilar $(40 \mu \mathrm{L})$ según las recomendaciones técnicas del fabricante para las concentraciones de glucosa en ayunas, los triglicéridos, el colesterol unido a las lipoproteínas de alta densidad y el colesterol total por técnicas enzimáticas con equipo portátil Cardiocheck ${ }^{\circledR}$. Las lipoproteínas de baja densidad se calcularon por la fórmula de Friedewald ${ }^{17}$ cuando los valores de los triglicéridos eran $\leq 400 \mathrm{mg} / \mathrm{dL}$. Las extracciones de sangre se realizaron entre las 07:00 y las 09:00, tras 10-12 horas de ayuno (promedio $\pm 11,2$ horas). 
Se calculó la puntuación de riesgo cardiovascular de manera continua (score de riesgo cardiometabólico), con la suma de los residuos tipificados (Z-score) de las variables de riesgo cardiovascular: el colesterol total, las lipoproteínas de baja densidad, los triglicéridos/lipoproteínas de alta densidad, la glucosa en ayunas y la tensión arterial media. La razón de probabilidad de incremento en el riesgo para enfermedad cerebrovascular, se obtuvo de los sujetos con valores $\geq 1 \pm \mathrm{DE}$ para la edad media como lo reporta el trabajo de Artero y Cols. ${ }^{18}$ Adicionalmente, se calcularon índices proaterogénicos: colesterol total/lipoproteínas de alta densidad $^{19}$, lipoproteínas de baja densidad/lipoproteínas de alta densidad, y triglicéridos/lipoproteínas de alta densidad como su expresión clínica ${ }^{20}$. Por último, se determinó la presión arterial con un esfingomanómetro digital Welch Allyn ${ }^{\circledR}$ modelo OSZ 5 (Illinois, EE.UU ${ }^{\circledR}$ ) en el brazo derecho en dos ocasiones, con un intervalo de cinco minutos entre sí, con los participantes en posición sedente y después de diez minutos de reposo. La tensión arterial media, se calculó mediante la fórmula: (2 • tensión arterial sistólica + Tensión arterial diastólica)/3.

\section{Componentes del síndrome metabólico}

Se tomaron los criterios descritos por la Federación Internacional de Diabetes ${ }^{7}$ : obesidad abdominal (circunferencia de cintura $\geq 90 \mathrm{~cm}$ (hombres) $\geq 80 \mathrm{~cm}$ (mujeres), $\mathrm{y} / \mathrm{o}$ triglicéridos $\geq 150 \mathrm{mg} / \mathrm{dL}$ y/o lipoproteínas de alta densi$\mathrm{dad}<40 \mathrm{mg} / \mathrm{dL}$ en hombres $\mathrm{y}<50$ en mujeres, y/o presión arterial $\geq 130 / 85 \mathrm{~mm} \mathrm{Hg}$, y/o glucosa plasmática en ayunas $\geq 110 \mathrm{mg} / \mathrm{dL}$.

\section{Biomarcadores de la función endotelial no invasiva}

La función endotelial se evaluó mediante la prueba de vasodilatación dependiente del endotelio medida por el flujo y la velocidad de onda de pulso. La primera, comprende la visualización de la arteria braquial, la medición de su diámetro y la velocidad del flujo sanguíneo, y tras un estímulo hiperérmico permite la liberación del óxido nítrico que puede ser cuantificado como indicativo de la función vasomotora, a través de las imágenes por pletismografía vascular con un equipo Toshiba Xario ${ }^{T M}$ con transductor lineal de $7,5 \mathrm{MHz}^{21}$. Los registros fueron obtenidos en formato Digital Imaging and Communication in Medicine y archivados en ese mismo formato. Tras cinco minutos de reposo, se ubicó al paciente en decúbito supino; en esta posición, se realizó la medición del calibre de la arteria braquial, un minuto antes y uno después de ocluirla con el brazalete del esfigmomanómetro durante cinco minutos (presión de $60 \mathrm{~mm} \mathrm{Hg}$ por encima de la presión arterial sistólica basal). Se colocó el transductor lineal a $60^{\circ}$ en relación con el eje longitudinal del

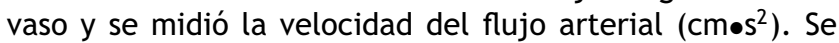
congeló la imagen cuando coincidió con el período diástole cardiaca y se identificó la interfase luz-íntima. Se midió el diámetro de la arteria braquial $(\mathrm{mm})$, y se marcó la piel del brazo para la colocación posterior del transductor (en la misma posición) para posteriores mediciones. La vasodilatación dependiente del endotelio se expresó como el porcentaje de cambio del diámetro arterial calculado con la fórmula vasodilatación dependiente del endotelio $=($ pico del diámetro hiperémico-diámetro arterial basal)/diámetro arterial basal $\times 100$. La segunda técnica empleada fue la medición de la velocidad de onda del pulso aórtica, con el equipo Arteriograph (TensioMed ${ }^{T M}$ ), instrumento validado de medición no invasiva, que permite evaluar la rigidez arterial a través de la velocidad de onda del pulso basándose en los datos oscilométricos obtenidos del brazo, mediante la oclusión de la arteria braquial con un manguito insuflado a una presión suprasistólica ${ }^{22}$. En la medida en que el tiempo de tránsito se hace más rápido está implícito el hecho de que hay una menor capacidad de distensión (mayor rigidez) de la aorta abdominal como el vaso de mayor calibre del cuerpo y, por ende, de las demás arterias. Con el paciente en posición supina, el operador medía la distancia entre la horquilla esternal y la sínfisis pubiana, que equivale a la distancia entre el cayado y la bifurcación de la aorta y colocaba el manguito rodeando el brazo izquierdo del paciente a $3 \mathrm{~cm}$ por arriba del pliegue del codo. La velocidad de onda del pulso indica el cociente entre la distancia recorrida por la onda del pulso (por la pared arterial) entre dos puntos del árbol vascular y el tiempo de tránsito. Ambas mediciones fueron realizadas por un operador ciego a los pacientes, previo entrenamiento en ambas técnicas.

\section{Intervención nutricional (estado de lipemia postprandial)}

El efecto de la ingesta alta en grasa sobre los biomarcadores de la función endotelial y metabólicos, se realizó tras un ayuno de 8-12 horas (valor basal, 0 minutos), y a los $60 \mathrm{~min}$., $120 \mathrm{~min}$., $180 \mathrm{~min}$. y $240 \mathrm{~min}$. postingesta, previa recomendación de una dieta estándar baja en nitratos 24 horas antes de las pruebas. Para inducir el estado de la lipemia postprandial se elaboró un menú que contenía 1.049 calorías, de los cuales $31 \mathrm{~g}$ provenían de proteínas, $79 \mathrm{~g}$ de grasas $(31 \mathrm{~g}$ de grasa saturada), $666 \mathrm{mg}$ de colesterol y $69 \mathrm{~g}$ de carbohidratos (software Ceres Versión 1.02, 1997, FAO). Un estudio piloto confirmó que esta ingesta producía alteración transitoria de la función endotelial ${ }^{3}$.

\section{Plan de análisis}

El procesamiento y análisis de la información se realizó con el programa Statistical Package for Social Science ${ }^{\circledR}$ software, versión 22 para Windows (SPSS; Chicago, IL, USA). Se analizó la normalidad de todas las variables, y en aquellas que no presentaron una distribución normal se realizó una transformación logarítmica (log). Los valores continuos se expresaron como media $( \pm$ ) desviación estándar. La comparación de medias se hizo con las pruebas ANOVA y ANCOVA ajustando por el sexo y la edad. Las diferencias entre las medias de cada grupo y momento de medición (0 min-basal y $60 \mathrm{~min}$., $120 \mathrm{~min}$., $180 \mathrm{~min}$. y $240 \mathrm{~min}$. postingesta) se analizaron mediante la corrección de Bonferroni para múltiples comparaciones (ANCOVA). La lipemia postprandial, se evaluó según la línea de Lairon y Cols. ${ }^{23}$ Para los triglicéridos y el colesterol total $(\mathrm{mg} / \mathrm{dL})$ se analizó el área bajo la curva con el método trapezoidal, llamado lipemia postprandial total o magnitud de lipemia postprandial o trigliceridemia postprandial. En ambas categorías del índice de masa corporal, se comparó el Z-score del riesgo cardiovascular entre la 
curva y una línea imaginaria trazada a partir de la concentración en ayunas en estado de trigliceridemia. En este caso no se realizó ningún ajuste, ya que este índice se estandarizó previamente para las variables de confusión. Al no encontrarse interacciones significativas en la variable sexo con otras variables del estudio, se realizaron los análisis para los hombres y las mujeres conjuntamente para lograr una mayor potencia estadística (ANCOVA). Por último, correlaciones parciales se estimaron con el cálculo del coeficiente de correlación de Pearson, entre el estado nutricional y los biomarcadores de la función endotelial y metabólicos, ajustados por la edad y el sexo. El nivel de significancia estadística se fijó a un valor $p<0,05$.

\section{Resultados}

Las características descriptivas de los participantes se presentan en la tabla 1. El análisis ANOVA mostró que las mujeres tenían valores de peso, altura, circunferencia de cintura mayores que los varones, mientras que éstos presentaban mayor porcentaje de grasa y unas lipoproteínas de alta densidad más saludables. En los biomarcadores de la función endotelial, los varones mostraron mayor porcentaje en el índice de aumentación aórtico que las mujeres, mientras que éstas mostraron cifras más altas del índice de aumentación braquial. No se encontraron diferencias significativas entre sexos en las otras características estudiadas.

Tabla 1 Características antropométricas, clínicas y función endotelial de la población evaluada

\begin{tabular}{|c|c|c|c|}
\hline Característica & Hombres $(n=22)$ & Mujeres $(n=11)$ & $\mathrm{p}$ \\
\hline \multicolumn{4}{|l|}{ Antropometría } \\
\hline Edad (años) & $31,3 \pm 7,7$ & $31,2 \pm 7,8$ & 0,962 \\
\hline Peso $(\mathrm{kg})$ & $64,7 \pm 10,1$ & $78,4 \pm 11,6$ & 0,001 \\
\hline Altura (m) & $1,61 \pm 0,05$ & $1,74 \pm 0,08$ & 0,001 \\
\hline Circunferencia de cintura $(\mathrm{cm})$ & $76,9 \pm 8,1$ & $88,6 \pm 10,6$ & 0,002 \\
\hline Circunferencia de cadera $(\mathrm{cm})$ & $101,6 \pm 6,9$ & $182,9 \pm 253,4$ & 0,135 \\
\hline Índice de masa corporal (kg/m²) & $25,0 \pm 3,5$ & $25,9 \pm 3,4$ & 0,528 \\
\hline \multicolumn{4}{|l|}{ Estado nutricional } \\
\hline Normopeso n (\%) & $9(40,9)$ & $13(59,1)$ & 0,809 \\
\hline Sobrepeso/obesidad n (\%) & $4(36,4)$ & $7(63,6)$ & 0,801 \\
\hline \multicolumn{4}{|l|}{ Composición corporal } \\
\hline RCE & $0,40 \pm 0,06$ & $0,74 \pm 0,91$ & 0,094 \\
\hline Masa grasa (kg) & $23,7 \pm 7,5$ & $20,0 \pm 8,9$ & 0,218 \\
\hline Grasa corporal (\%) & $35,6 \pm 6,5$ & $24,9 \pm 8,2$ & 0,001 \\
\hline \multicolumn{4}{|l|}{ Biomarcadores metabólicos } \\
\hline $\mathrm{CT}(\mathrm{mg} / \mathrm{dL})$ & $169,2 \pm 48,1$ & $159,6 \pm 30,6$ & 0,555 \\
\hline Triglicéridos (mg/dL) & $118,2 \pm 60,4$ & $115,6 \pm 51,1$ & 0,905 \\
\hline $\mathrm{HDL}(\mathrm{mg} / \mathrm{dL})$ & $46,9 \pm 9,8$ & $35,7 \pm 8,2$ & 0,003 \\
\hline LDL (mg/dL) & $102,2 \pm 39,0$ & $100,6 \pm 32,0$ & 0,910 \\
\hline Glucosa (mg/dL) & $83,1 \pm 11,3$ & $77,3 \pm 10,5$ & 0,164 \\
\hline Z-score del riesgo cardiometabólico & $-0,29 \pm 0,54$ & $-0,42 \pm 0,32$ & 0,493 \\
\hline $\mathrm{CT} / \mathrm{C}-\mathrm{HDL}$ & $3,8 \pm 1,5$ & $4,7 \pm 1,4$ & 0,100 \\
\hline C-LDL/C-HDL & $2,3 \pm 1,1$ & $3,0 \pm 1,2$ & 0,114 \\
\hline Log Triglicéridos/c-HDL & $0,8 \pm 0,6$ & $1,1 \pm 0,5$ & 0,192 \\
\hline \multicolumn{4}{|l|}{ Biomarcadores de función endotelial } \\
\hline VDE (\%) & $8,2 \pm 6,7$ & $4,2 \pm 3,1$ & 0,070 \\
\hline $\operatorname{VOP}(\mathrm{m} / \mathrm{s})$ & $6,9 \pm 0,9$ & $6,7 \pm 0,8$ & 0,581 \\
\hline Presión sistólica aórtica (mm Hg) & $102,7 \pm 35,3$ & $106,5 \pm 8,4$ & 0,732 \\
\hline Presión de pulso aórtica (mm Hg) & $44,1 \pm 17,4$ & $35,7 \pm 3,3$ & 0,123 \\
\hline Índice de aumentación braquial (\%) & $-27,2 \pm 23,0$ & $-49,9 \pm 13,5$ & 0,005 \\
\hline Índice de aumentación aórtico (\%) & $24,2 \pm 11,5$ & $12,4 \pm 6,8$ & 0,004 \\
\hline \multicolumn{4}{|l|}{ Presión arterial } \\
\hline TAS (mm Hg) & $118,2 \pm 6,0$ & $117,7 \pm 6,7$ & 0,830 \\
\hline TAD (mm Hg) & $72,2 \pm 7,3$ & $70,8 \pm 9,1$ & 0,634 \\
\hline TAM (mm Hg) & $87,6 \pm 5,8$ & $86,6 \pm 7,8$ & 0,654 \\
\hline
\end{tabular}

Valores presentados en media \pm desviación estándar. RCE: relación cintura/estatura. CT: colesterol total. c-HDL: colesterol unido a lipoproteínas de alta densidad. c-LDL: colesterol unido a lipoproteínas de baja densidad. VDE: vasodilatación dependiente del endotelio. VOP: velocidad de onda del pulso. TAS: tensión arterial sistólica. TAD: tensión arterial diastólica. TAM: tensión arterial media. El Z-score del riesgo cardiovascular se calculó a partir de la suma de los residuos tipificados (Z) de las variables del riesgo cardiovascular: CT, C-LDL, razón TG/c-HDL, glucosa y tensión arterial media. 
A.

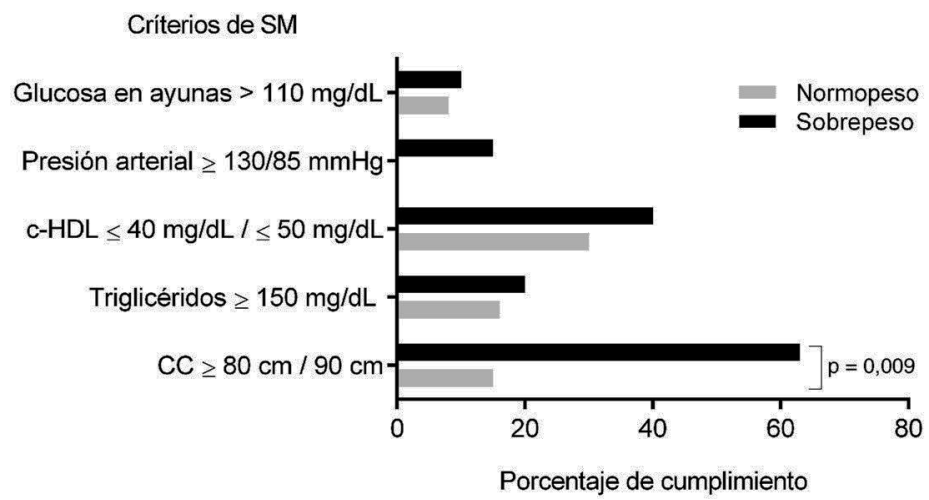

B.

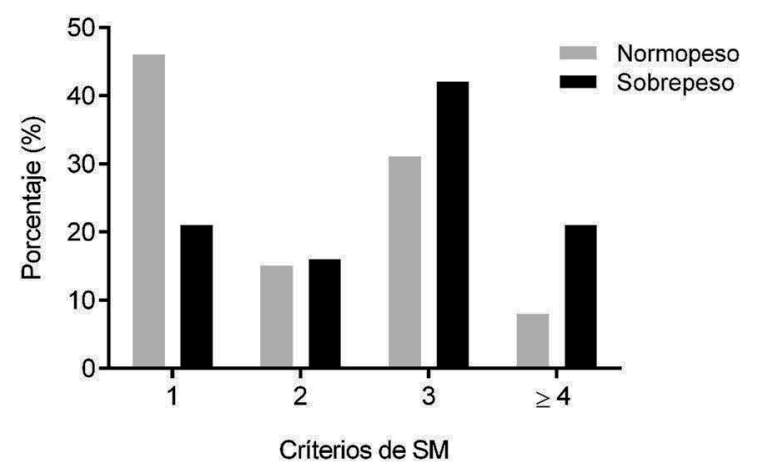

Figura 1 Porcentaje de cumplimiento (A) y criterios del síndrome metabólico (B) en función al estado nutricional.

El presente estudio detecta una prevalencia general del síndrome metabólico del $37 \%$ según criterios de la Federación Internacional de Diabetes. Al comparar por estado nutricional, la prevalencia del síndrome metabólico se incrementa a $42 \%$ en los sujetos con sobrepeso, mientras que en los participantes clasificados en normopeso, la prevalencia disminuye al $30 \%$. La obesidad abdominal, los bajos niveles de las lipoproteínas de alta densidad y la trigliceridemia, fueron los componentes con mayor prevalencia con valores de 63,40 y $20 \%$, respectivamente, en los sujetos con sobrepeso, mientras que la hipercolesterolemia (31\%) y los bajos niveles de las lipoproteínas de alta densidad (30\%), obtuvieron la mayor frecuencia en el grupo con normopeso (fig. 1A). En adición a lo anterior, los participantes con sobrepeso que sumaron 4 o más factores, mostraron $10 \%$ de mayor frecuencia de presentar criterios asociados al síndrome metabólico, (fig. 1B).

En la tabla 2 se muestran las diferencias metabólicas y la función endotelial según el estado nutricional. El análisis ANCOVA ajustado por sexo y edad, mostró que los sujetos clasificados en normopeso tenían menores cifras de circunferencia de cintura, relación cintura-estatura, masa- grasa, porcentaje de grasa total; así como un perfil cardiometabólico más saludable que su contraparte con sobrepeso. En este mismo grupo se muestran también los mayores valores en la velocidad de onda del pulso medida por oscilometría vascular.

Al estimar el efecto de la lipemia postprandial sobre los índices aterogénicos y el Z-score de riesgo cardiometabólico en función al peso, los participantes clasificados en sobrepeso muestran las diferencias significativas en los indicadores metabólicos Los triglicéridos/lipoproteínas de alta densidad (fig. $2 E$ ) entre el momento 0 min. y $240 \mathrm{~min}$. $(0,99 \pm 0,51$ vs. $1,80 \pm 0,63 \Delta=82 \%)$ y Z-score $(-0,288 \pm 0,391$ vs. $0,291 \pm 0,480 \Delta=201 \%)$, (fig. $2 \mathrm{G})$. En este mismo grupo, se observó un mayor nivel en el área bajo la curva, en la razón Log triglicéridos/lipoproteínas de alta densidad y en el Z-score del riesgo cardiometabólico (fig. $2 \mathrm{~F}$ y fig. 2H).

Se identificó que la lipemia postprandial reducía la vasodilatación dependiente del endotelio, aproximadamente, en $19,2 \%$ en los $60 \mathrm{~min}$. $(5,9 \pm 1,5 \%)$, y $240 \mathrm{~min}$. $(3,7 \pm 1,2 \%$, $\mathrm{p}<0,05)$, respectivamente (fig. $3 \mathrm{~A}$ y fig. $3 \mathrm{~B}$ ). Este hallazgo se acompañó de un incremento significativo en la velocidad de onda del pulso (fig. 3C), pasando de 6,9 $\pm 1,1 \mathrm{~m} / \mathrm{s}$ en estado basal, a 7,2 $\pm 1,2 \mathrm{~m} / \mathrm{s}$ a los $240 \mathrm{~min}$. postprandial $(p<0,05)$, resultado confirmado en el análisis del área bajo la curva-velocidad de onda del pulso $(6,4 \pm 0,2 \mathrm{~m} / \mathrm{s}$ vs. $7,0 \pm 0,1 \mathrm{~m} / \mathrm{s}, \mathrm{p}<0,001$ ) (fig. 3D).

Con el propósito de verificar si el estado nutricional se relacionaba con la velocidad de onda de pulso y con el área bajo la curva de los biomarcadores evaluados, se llevó a cabo un análisis de correlación con el coeficiente de Pearson ( $r$ ). Tras ajustar por edad y sexo, se observó en los sujetos con sobrepeso, una correlación entre la velocidad de onda del pulso y el área bajo la curva del Z-score de riesgo cardiometabólico $(r=0,287 p<0,01)$, además, de una correlación inversa entre la velocidad de onda del pulso y la vasodilatación dependiente del endotelio $(r=-0,125 p<0,01)$, tabla 3.

\section{Discusión}

Los principales resultados muestran que los sujetos clasificados en sobrepeso tenían un perfil cardiometabólico asociado con un mayor riesgo cardiovascular. Además, se pone de manifiesto que la lipemia postprandial y la disfunción endotelial son mayores en personas con sobrepeso que en aquellas con peso saludable. Se ha descrito que la alteración del metabolismo lipídico postprandial en personas con sobrepeso se puede explicar, por un lado, por la resistencia a la insulina asociada con la obesidad corporal o por el exceso de adiposidad en la región abdominal encontrada en este mismo grupo.

Existe evidencia que un incremento en el aclaramiento de los triglicéridos en el plasma puede ocasionar disfunción endotelial, hallazgo que fue corroborado en este estudio ${ }^{3}$. Sin embargo, existe muy poca información publicada referente al impacto de la lipemia postprandial sobre la rigidez arterial y la velocidad de onda del pulso ${ }^{24,25}$. Los trabajos de Daskalova y Cols. ${ }^{24}$ y Ding y Cols. ${ }^{25}$, muestran que un estado de hiperlipidemia e hiperglicemia, respectivamente, incrementan la rigidez vascular como consecuencia de un proceso de glicación que ocurre entre la glucosa y las proteínas de la matriz extracelular de la pared vascular. Otros autores ${ }^{3,26}$, 
Tabla 2 Características antropométricas, metabólicas y función endotelial según el estado nutricional

\begin{tabular}{lcc}
\hline Característica & $\begin{array}{c}\text { Normopeso } \\
(\mathrm{n}=13)\end{array}$ & $\begin{array}{l}\text { Sobrepeso } \\
(\mathrm{n}=20)\end{array}$ \\
\hline Antropometría & & \\
Circunferencia de cintura (cm) & $73,0 \pm 6,7$ & $85,6 \pm 9,3$ \\
RCE & $0,45 \pm 0,04$ & $0,60 \pm 0,86$ \\
Masa grasa (kg) & $26,3 \pm 7,2$ & $35,7 \pm 7,5$ \\
Grasa corporal (\%) & $15,7 \pm 4,9$ & $26,8 \pm 6,5$ \\
Biomarcadores metabólicos & & \\
CT (mg/dL) & $170,1 \pm 41,7$ & 0,001 \\
Triglicéridos (mg/dL) & $114,7 \pm 62,7$ & 0,001 \\
HDL (mg/dL) & $46,8 \pm 12,7$ & $163,4 \pm 44,4$ \\
LDL (mg/dL) & $100,2 \pm 41,5$ & $119,1 \pm 54,0$ \\
Glucosa (mg/dL) & $77,8 \pm 10,8$ & $40,9 \pm 8,4$ \\
Z-score del riesgo cardiometabólico & $-0,41 \pm 0,59$ & $102,6 \pm 33,7$ \\
CT/c-HDL & $4,0 \pm 1,9$ & $83,3 \pm 11,2$ \\
C-LDL/c-HDL & $2,5 \pm 1,5$ & $-0,29 \pm 0,39$ \\
Log Triglicéridos/c-HDL & $0,8 \pm 0,6$ & $4,1 \pm 1,3$ \\
Biomarcadores de función endotelial & & $2,6 \pm 0,9$ \\
VDE (\%) & $7,3 \pm 5,2$ & 0,833 \\
VOP (m/s) & $6,4 \pm 0,8$ & 0,118 \\
Presión sistólica aórtica (mm Hg) & $105,9 \pm 6,6$ & 0,858 \\
Presión de pulso aórtica (mm Hg) & $38,1 \pm 4,8$ & 0,176 \\
Índice de aumentación braquial (\%) & $-43,3 \pm 15,5$ & 0,010 \\
Índice de aumentación aórtico (\%) & $15,7 \pm 7,9$ & 0,840 \\
\hline
\end{tabular}

Valores presentados en media \pm desviación estándar. RCE: relación cintura/estatura; CT: colesterol total; c-HDL: colesterol unido a lipoproteínas de alta densidad; c-LDL: colesterol unido a lipoproteínas de baja densidad; VDE: vasodilatación dependiente del endotelio; VOP: velocidad de onda de pulso; TAS: tensión arterial sistólica; TAD: tensión arterial diastólica; TAM: tensión arterial media. El Z-score de riesgo cardiovascular se calculó a partir de la suma de los residuos tipificados (Z) de las variables de riesgo cardiovascular: CT, C-LDL, razón TG/c-HDL, glucosa y tensión arterial media.

Tabla 3 Correlaciones parciales entre la velocidad de onda de pulso y el área bajo la curva de los biomarcadores metabólicos en función al estado nutricional

\begin{tabular}{llc}
\hline Característica & $\begin{array}{l}\text { Normopeso } \\
(\mathrm{n}=13)\end{array}$ & $\begin{array}{l}\text { Sobrepeso } \\
(\mathrm{n}=20)\end{array}$ \\
\hline ABC CT/C-HDL & 0,119 & 0,181 \\
ABC c-LDL/c-HDL & 0,139 & 0,203 \\
ABC Log Triglicéridos/c-HDL & 0,096 & 0,231 \\
$\begin{array}{l}\text { ABC Z-score del riesgo } \\
\quad \text { cardiometabólico }\end{array}$ & 0,133 & $0,287^{*}$ \\
VDE (\%) & 0,051 & $-0,125^{*}$ \\
\hline
\end{tabular}

CT: colesterol total. c-HDL: colesterol unido a lipoproteínas de alta densidad. C-LDL: colesterol unido a lipoproteínas de baja densidad. VDE: vasodilatación dependiente del endotelio.

El Z-score del riesgo cardiovascular se calculó a partir de la suma de los residuos tipificados $(Z)$ de las variables del riesgo cardiovascular: $C T, c-L D L$, razón TG/c-HDL, glucosa y tensión arterial media.

* $\mathrm{p}<0,01$ por correlación Pearson. $\mathrm{ABC}$ : área bajo la curva.

han descrito que los ácidos grasos libres pueden conducir a disfunción endotelial por el incremento en la producción del anión súperoxido $\left(\mathrm{O}_{2}^{-}\right)$, y una subsiguiente disminución de la biodisponibilidad del óxido nítrico, como se observó a los $180 \mathrm{~min}$. y $240 \mathrm{~min}$. postprandiales o por el incremento en la velocidad de onda del pulso pasando de $6,9 \pm 1,1 \mathrm{~m} / \mathrm{s}$ en estado basal, a 7,2 $\pm 1,2 \mathrm{~m} / \mathrm{s}$ a los $240 \mathrm{~min}$. postprandial $(p<0,05)$, resultado confirmado en el análisis del área bajo la curva (fig. 3D). Recientemente, se demostró que sujetos con exceso de peso que ingieren dietas altas en grasas, presentan menor función endotelial y mayores concentraciones de triglicéridos postprandiales, marcadores que también se han descrito en pacientes con la enfermedad cerebrovascular relacionadas con la resistencia a la insulina ${ }^{27}$. Así, la obesidad, y sobre todo el depósito de grasa visceral, expresado como la circunferencia de la cintura, son situaciones que conducen a disfunción endotelial y a un metabolismo inadecuado de los lípidos en situación postprandial, aspecto corroborado en este estudio ${ }^{3,9}$.

El presente estudio detecta una prevalencia del síndrome metabólico del $37 \%$ según los criterios de la Federación Internacional de Diabetes ${ }^{7}$, resultado superior al encontrado en los estudios de Ruiz y Cols. ${ }^{28}(9 \%)$, Villegas y Cols. ${ }^{29}(23 \%)$ y Fonseca-Camacho y Cols. ${ }^{30}(23 \%)$ en la población con características similares a las de este estudio. Respecto a los componentes del síndrome metabólico (fig. 1A), la obesidad abdominal (63\%), los bajos niveles de las lipoproteínas de alta densidad (40\%) y la trigliceridemia (20\%), fueron los componentes con mayor prevalencia, resultado mayor al descrito por Villegas y Cols. ${ }^{29}$ (28\%) en el componente de la obesidad central y $(39 \%)$ en los bajos niveles de las lipoproteínas de alta densidad. Estos hallazgos también son mayores a la media ponderada del Consenso Latinoamericano de Hipertensión en pacientes con diabetes tipo 2 y síndrome metabólico (24,9\% rango: $18,8-43,3 \%)$, menor al 
a.
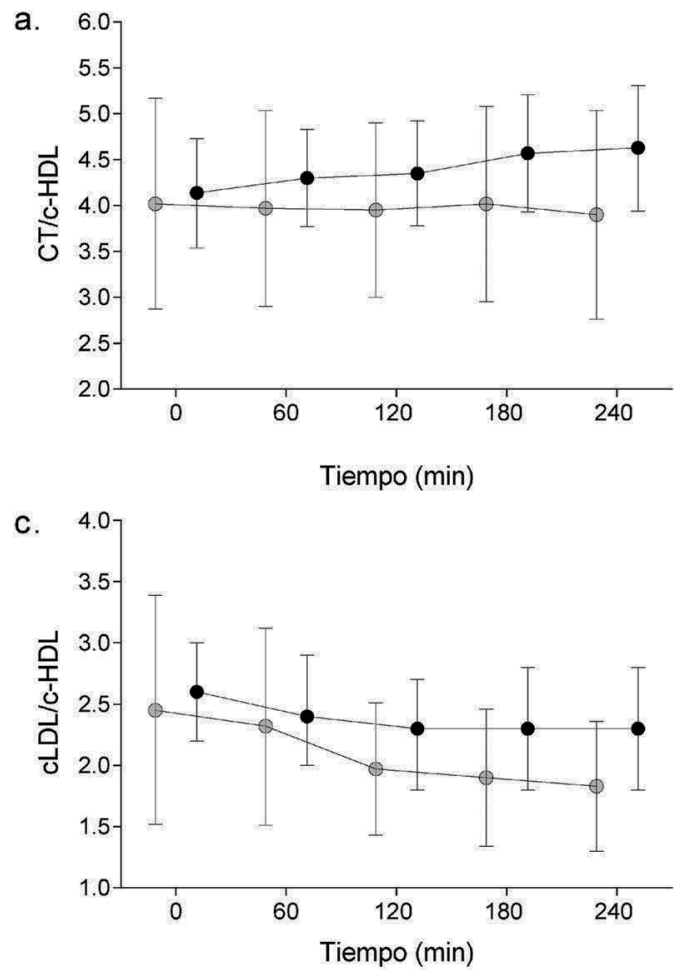

Sobrepeso

- Normopeso

Sobrepeso Normopeso

d.
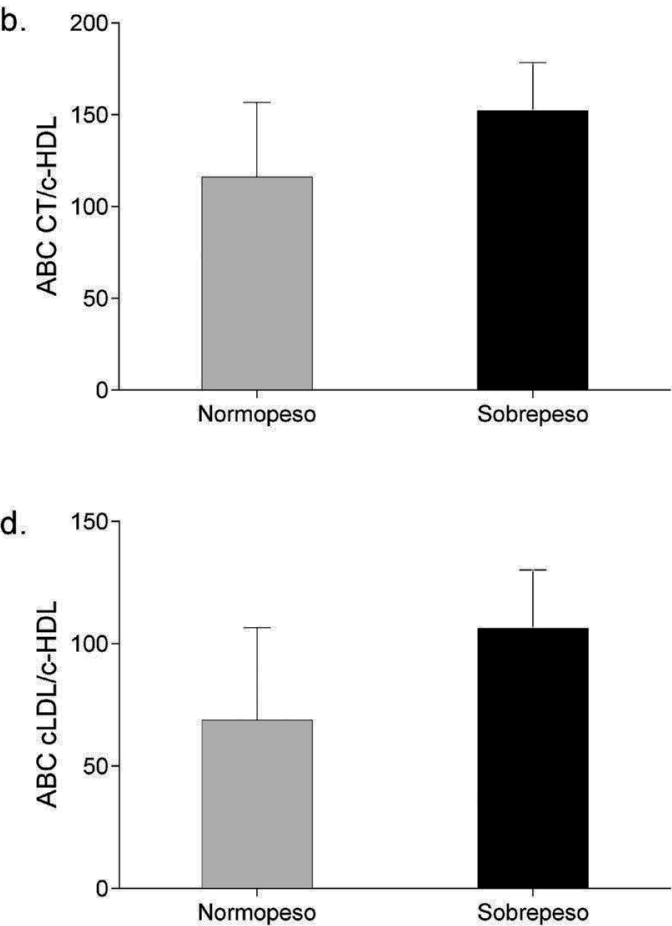
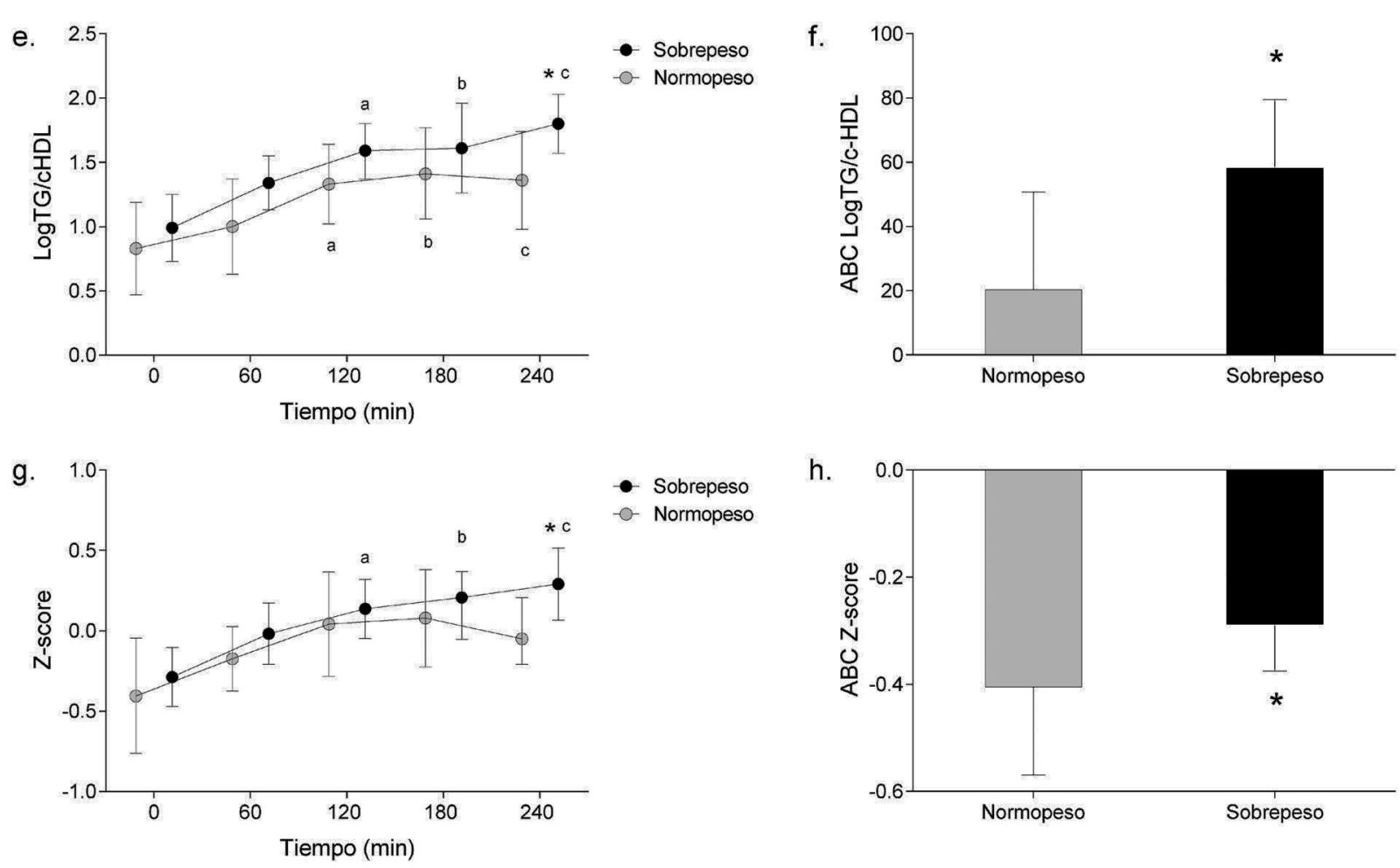

h.

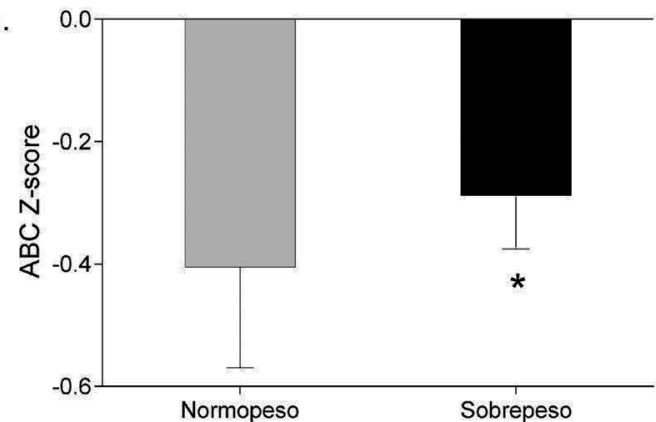

Figura 2 Efecto de la lipemia postprandial sobre índices aterogénicos y Z-score del riesgo cardiometabólico en función al estado nutricional de sujetos con criterios del síndrome metabólico. ${ }^{a}$ Relación CT/c-HDL. ${ }^{b}$ Área bajo la curva de la Relación CT/c-HDL. ${ }^{c}$ Relación c-LDL/c-HDL. ${ }^{d}$ Área bajo la curva de la Relación c-LDL/c-HDL. ${ }^{e}$ Relación log TG/c-HDL. ${ }^{f}$ Área bajo la curva de la Relación log TG/c-HDL. ${ }^{\mathrm{g}}$ Z-score de síndrome metabólico. ${ }^{\mathrm{h}}$ Área bajo la curva del Z-score de síndrome metabólico. CT: colesterol total; c-HDL: colesterol unido a lipoproteínas de alta densidad; c-LDL: colesterol unido a lipoproteínas de baja densidad; ABC: área bajo la curva. El Z-score de riesgo cardiovascular se calculó a partir de la suma de los residuos tipificados (Z) de las variables de riesgo cardiovascular: CT: c-LDL; razón TG/c-HDL: glucosa y tensión arterial media. a Diferencia entre 0 min vs. 120 min. ${ }^{b}$ Diferencia entre 0 min vs. 180 min. c Diferencia entre 0 min vs. 240. * Diferencias en función al estado nutricional. $p<0,05$. 

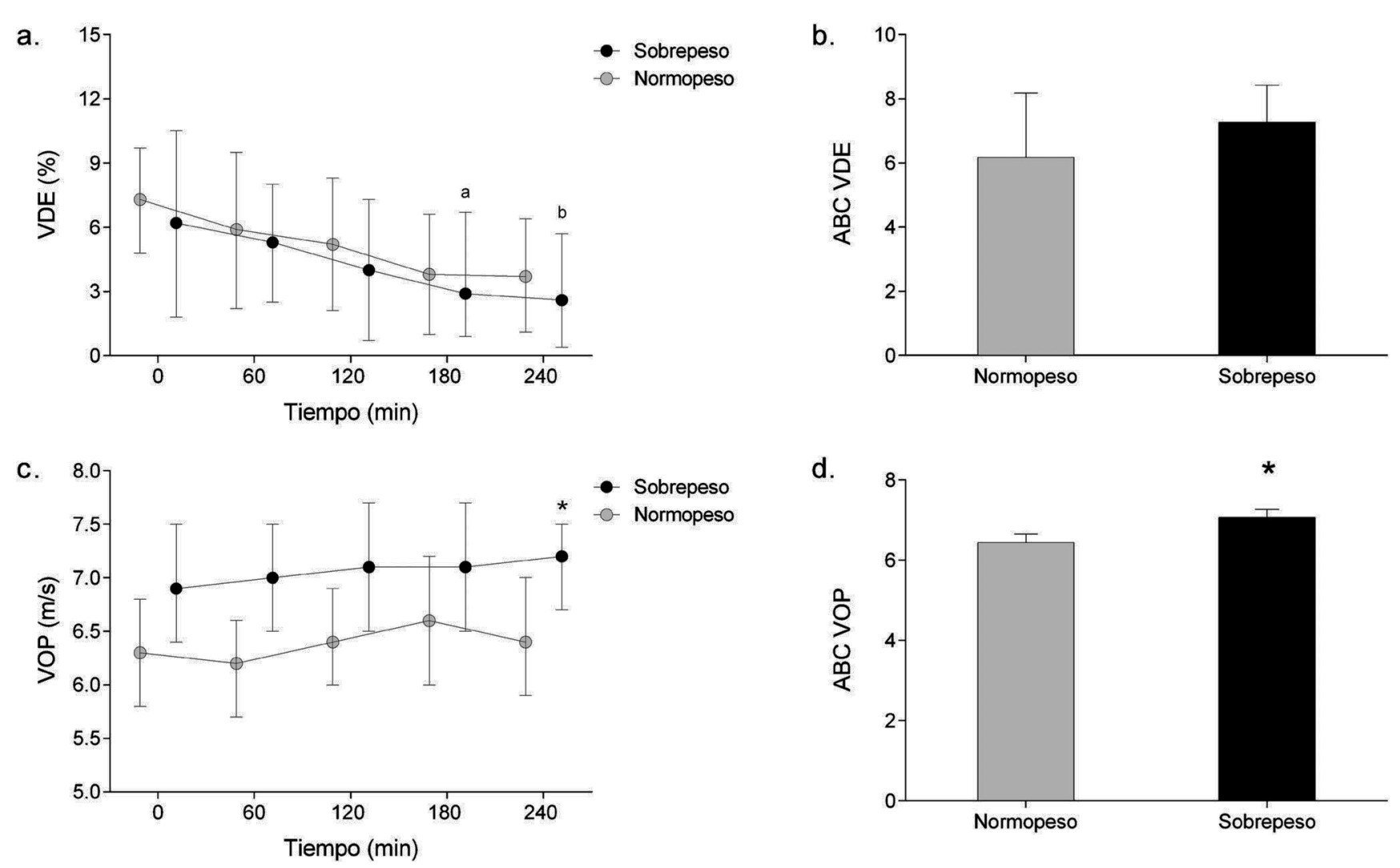

Figura 3 Efecto de la lipemia postprandial en los biomarcadores de la función endotelial según el estado nutricional de sujetos con criterios del síndrome metabólico. ${ }^{a}$ Vasodilatación dependiente del endotelio. ${ }^{\text {b }}$ Área bajo la curva de la vasodilatación dependiente del endotelio. ' Velocidad de la onda de pulso. ' Área bajo la curva de la velocidad de la onda de pulso. VDE: vasodilatación dependiente del endotelio. VDE: vasodilatación dependiente del endotelio; VOP: velocidad de onda de pulso; ABC: área bajo la curva. ${ }^{a}$ Diferencia entre 0 min vs. 180 min. ${ }^{\text {b }}$ Diferencia entre 0 min vs. 240 . * Diferencias en función al estado nutricional. p < 0,05 .

componente (lipoproteínas de alta densidad bajo 62,9\%) y mayor que la obesidad abdominal $(45,8 \%)^{31}$.

En estudios poblacionales estadounidenses, con sujetos de edades similares a los presentados en este trabajo, la prevalencia del síndrome metabólico fue de $(24 \%)^{32}$ y en la población española de $(13,4 \%)^{33}$. Al diferenciar por subgrupos, los sujetos con sobrepeso presentaron mayor frecuencia de componentes del síndrome metabólico (fig. 1B), siendo la obesidad central el criterio de mayor prevalencia (63\%). En estudios epidemiológicos prospectivos, se ha constatado el aumento en la prevalencia de la obesidad abdominal en la última década con tasas del 42 y $50 \%$ en hombres de Norteamérica ${ }^{34}$ y latinos $^{35}$, respectivamente. Previamente, se ha identificado que el exceso de tejido adiposo a nivel central, representan alrededor del 50\% del riesgo atribuible poblacional para el desarrollo de enfermedad cardiocerebrovascular ${ }^{36}$

En complemento a lo anterior, el análisis ANCOVA ajustando por el sexo y la edad, mostró que los sujetos clasificados en normopeso tenían menores cifras de circunferencia de la cintura, relación cintura-estatura, masa grasa, porcentaje de grasa total; así como un perfil cardiometabólico más saludable que su contraparte con sobrepeso. En este mismo grupo se muestran también mayores valores en la velocidad de onda del pulso medida por oscilometría vascular. En los varones canadienses, Blackburn y Cols. ${ }^{37}$, demostraron también que la obesidad y sobre todo la acumulación de grasa abdominal alteran la lipemia postprandial. En esta misma línea, González y Cols. ${ }^{9}$, demostraron que los triglicéridos en la sangre capilar dependen de la obesidad abdominal y de los valores plasmáticos de los triglicéridos en ayunas. Estos autores, compararon los valores de los triglicéridos en la sangre capilar entre varones con obesidad moderada y con peso normal (tomando como obesidad moderada valores del índice de masa corporal comprendidos entre 25 y $30 \mathrm{~kg} / \mathrm{m}^{2}$ ) observando diferencias en los valores de los triglicéridos en ayunas entre ambos grupos, junto a una respuesta de lipemia postprandial, superior en los participantes con sobrepeso. Otros estudios han puesto de manifiesto que las personas obesas tienen una lipemia postprandial elevada. Así, Van Wijk y Cols. ${ }^{10}$, al estudiar la lipemia postprandial en sujetos con exceso de peso, observaron que el perfil diurno de los triglicéridos en la sangre capilar fue mayor en sobrepeso, sin diferencias entre los diabéticos y los no diabéticos. En este sentido, nuestros resultados concuerdan con esta hipótesis, pues muestran que las personas con exceso de peso, presentan valores significativamente mayores del área bajo la curva - Log triglicéridos/lipoproteínas de alta densidad, el área bajo la curva del Z-score del riesgo cardiometabólico y la disfunción 
endotelial. Con base a lo anterior, varios estudios han sugerido que los niveles de Log triglicéridos/lipoproteínas de alta densidad postprandiales pueden estar estrechamente asociados con el riesgo aterogénico de los valores plasmáticos de los triglicéridos ${ }^{25-38}$. Los individuos con elevados cociente Log triglicéridos/lipoproteínas de alta densidad o lipoproteínas de baja densidad/lipoproteínas de alta densidad, tienen un riesgo cardiovascular mayor, debido a la existencia de un desequilibrio entre el colesterol vehiculizado por las lipoproteínas aterogénicas y las lipoproteínas protectoras. Este desequilibrio puede deberse a un incremento de las variables aterogénicas contenidas en el numerador, a un descenso de la variable antiaterogénica del denominador o a ambos ${ }^{39}$.

Como análisis complementario en nuestro estudio, se valoró el grado de asociación entre la velocidad de onda del pulso y el área bajo la curva de distintos biomarcadores metabólicos en función al estado nutricional. Los resultados muestran que los participantes con sobrepeso muestran valores menos desfavorables en la circunferencia de la cintura, la grasa corporal, el Z- score de riesgo cardiometabólico y la velocidad de onda del pulso. En este mismo grupo, se observó un mayor nivel en el área bajo la curva en la razón Log triglicéridos/lipoproteínas de alta densidad y en el Z-score de riesgo cardiometabólico, confirmando la relevancia de la obesidad con el incremento en el riesgo cardiovascular de adultos normolipídicos.

Puesto que pasamos la mayoría de nuestro tiempo diario en el estado postprandial, los resultados de este estudio indican la necesidad de tener en cuenta la reducción de los lípidos en la dieta como estrategia adicional en la prevención del desarrollo prematuro del riesgo cardiovascular, además, de la inclusión de cambios en el estilo de vida ${ }^{40}$. Sin embargo, se resalta que en este estudio fue usada una dieta "experimental" que contenía $79 \mathrm{~g}$ de grasas (31 g de grasa saturada), la cual sobrepasa 3 veces la recomendación máxima nutricional. La dieta rica en grasa saturada usada en este estudio provoca una mayor respuesta de las lipoproteínas ricas en triglicéridos con persistencia de los quilomicrones, con la consecuente disfunción endotelial. Sobre este particular, los estudios de Patsch en el año 1992, fueron los primeros en sugerir que las concentraciones de triglicéridos en el plasma en el período postprandial, son marcadores independientes de la enfermedad cerebrovascular ${ }^{41}$, hallazgo que coincide con este reporte de investigación. Así, estos datos apoyan la posibilidad de incluir la lipemia postprandial como otra característica del estado nutricional y del riesgo cardiometabólico.

Las principales limitaciones del presente estudio son las inherentes a su carácter transversal. Si bien el número de pacientes incluidos en nuestro estudio fue relativamente pequeño, representando una eventual limitación, los pocos estudios publicados hasta la fecha sobre este tema en particular, son aún más pequeños y carecen de datos regionales. Adicionalmente, no se tuvo en cuenta los patrones de alimentación y de la actividad física de los sujetos que pueden modular la respuesta metabólica y vascular. Por último, la utilización de un Z-score de riesgo cardiometabólico como herramienta para evaluar globalmente el metabolismo de los lípidos y de los hidratos de carbono, puede inducir sesgos, pues las concentraciones de los triglicéridos se distribuyen de forma no gaussiana en cualquier población ${ }^{42}$.
No obstante, en este estudio se aplicó la transformación logarítmica triglicéridos/lipoproteínas de alta densidad para disminuir dicha variabilidad a la suma de los valores tipificados.

\section{Conclusiones}

Los resultados de este estudio indican que los adultos con criterios del síndrome metabólico clasificados en sobrepeso, muestran un perfil cardiometabólico asociado con un mayor riesgo cardiovascular. Además, se pone de manifiesto que la lipemia postprandial y la disfunción endotelial son mayores en personas con sobrepeso que en aquellas con peso saludable. En un futuro será necesario establecer el papel de la lipemia postprandial como factor de riesgo cardiovascular en especial en sujetos con exceso de peso.

\section{Colaboradores}

RR-V, JE-CB y DMD-V trabajó en la concepción, diseño del estudio, en el análisis de los resultados al igual que en la escritura del manuscrito y aprobación de la versión final. JCP, LAT-T, DC-P, ES-A y AT-S trabajaron en el diseño del estudio, en la recolección y el análisis de la información, en la redacción y aprobación de la versión final. PAH-Q, RR$\mathrm{V}, \mathrm{JE}-\mathrm{CB}$ y $\mathrm{DMD}-\mathrm{V}$ trabajaron en el análisis de los resultados y aprobación de la versión final.

\section{Responsabilidades éticas}

Protección de personas y animales. Los autores declaran que los procedimientos seguidos se conformaron a las normas éticas del comité de experimentación humana responsable y de acuerdo con la Asociación Médica Mundial y la Declaración de Helsinki.

Confidencialidad de los datos. Los autores declaran que han seguido los protocolos de su centro de trabajo sobre la publicación de datos de pacientes.

Derecho a la privacidad y consentimiento informado. Los autores han obtenido el consentimiento informado de los pacientes y/o sujetos referidos en el artículo. Este documento obra en poder del autor de correspondencia.

\section{Conflicto de intereses}

Los autores declaran no tener ningún conflicto de intereses.

\section{Agradecimientos}

Los autores quieren expresar su agradecimiento a todos los funcionarios de la Universidad del Rosario que participaron en este estudio.

\section{Bibliografía}

1. Pirillo A, Norata GD, Catapano AL. Postprandial lipemia as a cardiometabolic risk factor. Curr Med Res Opin. 2014;30:1489-503. 
2. Chan DC, Pang J, Romic G, Watts GF. Postprandial hypertriglyceridemia and cardiovascular disease: current and future therapies. Curr Atheroscler Rep. 2013;15:309.

3. Ramírez-Vélez R. Postprandial lipemia induces endothelial dysfunction and higher insulin resistance in healthy subjects. Endocrinol Nutr. 2011;58:529-35.

4. Mah E, Noh SK, Ballard KD, Matos ME, Volek JS, Bruno RS. Postprandial hyperglycemia impairs vascular endothelial function in healthy men by inducing lipid peroxidation and increasing asymmetric dimethylarginine: arginine. J Nutr. 2011;141:1961-8.

5. Mozaffarian D, Aro A, Willett WC. Health effects of trans-fatty acids: experimental and observational evidence. Eur J Clin Nutr. 2009;63:S5-21.

6. Stamler J, Daviglus ML, Garside DB, Dyer AR, Greenland P, Neaton JD. Relationship of baseline serum cholesterol levels in 3 large cohorts of younger men to long-term coronary, cardiovascular, and all-cause mortality and to longevity. JAMA. 2000;284:311-8.

7. Alberti KG, Eckel RH, Grundy SM, Zimmet PZ, Cleeman JI, Donato KA, et al. Harmonizing the metabolic syndrome: a joint interim statement of the International Diabetes Federation Task Force on Epidemiology and Prevention; National Heart, Lung, and Blood Institute; American Heart Association; World Heart Federation; International Atherosclerosis Society; and International Association for the Study of Obesity. Circulation. 2009;120:1640-5.

8. Nogaroto V, Rodrigues MR, Vicari MR, De Almeida MC, Milléo FQ, Dos Santos FA, et al. High postprandial triglycerides serum levels: is obesity a good predictor. An Acad Bras Cienc. 2015;87:437-45.

9. González C, Real JT, Bartual A, Chaves FJ, García García AB, Blesa S, et al. Determinantes de la lipemia posprandial medida como perfil diurno de triglicéridos en personas no diabéticas con normolipemia. Med Clin (Barc). 2005;125:448-52.

10. Van Wijk JP, Halkes CJ, Erkelens DW, Castro Cabezas M. Fasting and daylong triglycerides in obesity with and without type 2 diabetes. Metabolism. 2003;52:1043-9.

11. De Oya M. Lipemia posprandial y obesidad. Med Clin (Barc). 2005;125:458-9.

12. de Vries MA, Klop B, Alipour A, van de Geijn GJ, Prinzen L, Liem $\mathrm{AH}$, et al. In vivo evidence for chylomicrons as mediators of postprandial inflammation. Atherosclerosis. 2015;243:540-5.

13. Delawi D, Meijenssen S, Castro Cabezas M. Intra-individual variations of fasting plasma lipids, apolipoproteins and postprandial lipemia in familial combined hyperlipidemia compared to controls. Clin Chim Acta. 2003;328:139-45.

14. Popkin BM. Global nutrition dynamics: the world is shifting rapidly toward a diet linked with noncommunicable diseases. Am J Clin Nutr. 2006;84:289-98.

15. López CA, Ramírez-Vélez R, Gallardo CEG, Marmolejo LC. Características morfofuncionales de individuos físicamente activos. latreia. 2008;21:121-8.

16. World Health Organization. Obesity: preventing and managing the global epidemic. Report of a WHO Consultation. World Health Organ Tech Rep Ser. 2000;894:1-255.

17. Friedewald WT, Levy IR, Fredrickson DS. Estimation of the concentration of low density lipoprotein cholesterol in plasma, without use of preparative ultracentrifuge. Clin Chem. 1972;18:499-502.

18. Artero EG, Ruiz JR, Ortega FB, España-Romero V, VicenteRodríguez G, Molnar D, et al. Muscular and cardiorespiratory fitness are independently associated with metabolic risk in adolescents: the HELENA study. Pediatric Diabetes. 2011;12:704-12.

19. Millán J, Pintó X, Múñoz A, Zúñiga M, Rubiés-Prat J, Pallardo LF, et al. Lipoprotein ratios: Physiological significance and clinical usefulness in cardiovascular prevention. Vasc Health Risk Manag. 2009;5:757-65.
20. Cordero A, Andrés E, Ordóñez B, León M, Laclaustra M, Grima $A$, et al. Usefulness of triglycerides-to-high-density lipoprotein cholesterol ratio for predicting the first coronary event in men. Am J Cardiol. 2009;104:1393-7.

21. Corretti MC, Anderson TJ, Benjamin EJ. Guidelines for the ultrasound assessment of endothelial-dependent flow-mediated vasodilation of the brachial artery: a report of the International Brachial Artery Reactivity Task Force. J Am Coll Cardiol. 2002;39:257-65.

22. Stoner L, Young JM, Fryer S. Assessments of arterial stiffness and endothelial function using pulse wave analysis. Int J Vasc Med. 2012;2012:903107.

23. Lairon D, López-Miranda J, Williams C. Methodology for studying postprandial lipid metabolism. Eur J Clin Nutr. 2007;61:1145-61.

24. Daskalova DC, Kolovou GD, Panagiotakos DB, Pilatis ND, Cokkinos DV. Increase in aortic pulse wave velocity is associated with abnormal postprandial triglyceride response. Clin Cardiol. 2005;28:577-83.

25. Ding C, Hsu SH, Wu YJ1, Su TC. Additive effects of postchallenge hyperglycemia and low-density lipoprotein particles on the risk of arterial stiffness in healthy adults. Lipids Health Dis. 2014;13:179.

26. Ramírez-Vélez R, Ortega JG, Hurtado C, Agredo-Zuñiga RA. Endothelial function is affected by postprandial lipemia: presentation of two cases and literature review. Med UIS. 2011;24:87-93.

27. Borén J, Matikainen N, Adiels M, Taskinen MR. Postprandial hypertriglyceridemia as a coronary risk factor. Clin Chim Acta. 2014;431:131-42.

28. Ruiz ÁJ, Aschner PJ, Puerta MF, Cristancho RA. IDEA study (International Day for the Evaluation of Abdominal Obesity): primary care study of the prevalence of abdominal obesity and associated risk factors in Colombia. Biomdica. 2012;32:610-6.

29. Villegas A, Botero J, Arango I, Arias S, Toro M. Prevalencia del síndrome metabólico en El Retiro, Colombia. Iatreia. 2003:291-7.

30. Fonseca-Camacho DF, Hernández-Fonseca JM, González-Ruíz K, Tordecilla-Sanders A, Ramírez-Vélez R. A better self-perception of physical fitness is associated with lower prevalence of metabolic syndrome and its components among university students. Nutr Hosp. 2014;31:1254-63.

31. López-Jaramillo P, Sánchez RA, Díaz M, Cobos L, Bryce A, ParraCarrillo JZ, En nombre del Grupo de Expertos de Latinoamérica. Latin American consensus on hypertension in patients with diabetes type 2 and metabolic syndrome. Clin Investig Arterioscler. 2014;26:85-103.

32. Ford ES, Giles WH, Dietz WH. Prevalence of the metabolic syndrome among US adults: findings from the third National Health and Nutrition Examination Survey. JAMA. 2002;287:356-9.

33. León Latre M, Andrés EM, Cordero A, Pascual I, Vispe C, Laclaustra $M$, et al. Relación entre el síndrome metabólico y la mortalidad por cardiopatía isquémica en España. Rev Esp Cardiol. 2009;62:1469-72.

34. Li C, Ford ES, MCGuire LC, Mokdad AH. Increasing trends in waist circumference and abdominal obesity among US adults. Obesity (Silver Spring). 2007;15:216-24.

35. Aschner P, Buendía R, Brajkovich I, González A, Figueredo R, Juárez XE, Uriza F, et at. Determination of the cutoff point for waist circumference that establishes the presence of abdominal obesity in Latin American men and women. Diabetes Res Clin Pract. 2011;93:243-7.

36. O’Donnell MJ, Xavier D, Liu L, INTERSTROKE investigators. Risk factors for ischaemic and intracerebral haemorrhagic stroke in 22 countries (the INTERSTROKE study): a case-control study. Lancet. 2010;376:112-23.

37. Blackburn P, Lamarche B, Couillard C, Pascot A, Bergeron N, Prud'homme D, et al. Postprandial hyperlipidemia: another 
correlate of the «hypertriglyceridemic waist» phenotype in men. Atherosclerosis. 2003;171:327-36.

38. Nagata T, Sugiyama D, Kise T, Tsuji S, Ohira H, Sato I, et al. Fasting remnant lipoproteins can predict postprandial hyperlipidemia. Lipids Health Dis. 2012;11:146.

39. Millán J, Pintó X, Múñoz A, Zúñiga M, Rubiés-Prat J, Pallardo LF, et al. Cocientes lipoproteicos: significado fisiológico y utilidad clínica de los índices aterogénicos en prevención cardiovascular. Clin Invest Arterioscl. 2010;22:25-32.
40. Pearson T. Cardiovascular Update: Risk, Guidelines, and Recommendations. Workplace Health Saf. 2015;63:376-80.

41. Patsch JR, Miesenböck G, Hopferwieser T, Mühlberger V, Knapp E, Dunn JK, et al. Relation of triglyceride metabolism and coronary artery disease. Studies in the postprandial state. Arterioscler Thromb. 1992;12:1336-45.

42. Carreras-González G, Ordóñez-Llanos J. Adolescence, physical activity, and metabolic cardiovascular risk factors. Rev Esp Cardiol. 2007;60:565-8. 